\title{
Characterization of Skew CR-Warped Product Submanifolds in Complex Space Forms via Differential Equations
}

\author{
Ibrahim Al-Dayel ${ }^{1}$ and Meraj Ali Khan $\mathbb{D}^{2}$ \\ ${ }^{1}$ Department of Mathematics and Statistics, College of Science, Imam Mohammad Ibn Saud Islamic University, \\ Riyadh 11566, Saudi Arabia \\ ${ }^{2}$ Department of Mathematics, College of Science, University of Tabuk, Tabuk, Saudi Arabia \\ Correspondence should be addressed to Meraj Ali Khan; meraj79@gmail.com
}

Received 14 April 2021; Accepted 19 June 2021; Published 6 July 2021

Academic Editor: Bosheng Song

Copyright (c) 2021 Ibrahim Al-Dayel and Meraj Ali Khan. This is an open access article distributed under the Creative Commons Attribution License, which permits unrestricted use, distribution, and reproduction in any medium, provided the original work is properly cited.

\begin{abstract}
Recently, we have obtained Ricci curvature inequalities for skew CR-warped product submanifolds in the framework of complex space form. By the application of Bochner's formula on these inequalities, we show that, under certain conditions, the base of these submanifolds is isometric to the Euclidean space. Furthermore, we study the impact of some differential equations on skew CR-warped product submanifolds and prove that, under some geometric conditions, the base is isometric to a special type of warped product.
\end{abstract}

\section{Introduction}

The studies $[1,2]$ provide both important intrinsic geometric as well as isometric properties of Riemannian manifolds via differential equations. It is well known that the classification of differential equations has a significant effect on the global study of Riemannian manifolds. In 1978, Tanno [2] studied various aspects of differential equations on Riemannian manifolds. In particular, the authors of $[3,4]$ characterized Euclidean sphere by the approach of differential equations. The analysis performed in $[2,5]$ proved that a nonconstant function $\lambda$ on a complete Riemannian manifold $\left(U^{n}, g\right)$ satisfies the differential equation

$$
\nabla^{2} \lambda+c g=0
$$

if and only if $\left(U^{n}, g\right)$ is isometric to Euclidean space $R^{n}$, where $c$ is constant.

Moreover, Garcia-Rio et al. [4] proved that, under some restrictions, the Riemannian manifold is isometric to warped product $U \times{ }_{f} R$, where $U$ is a complete Riemannian manifold, $R$ is the Euclidean line, and $f$ is the warping function. Moreover, warping function $f$ satisfies the second-order differential equation

$$
\frac{\mathrm{d}^{2} f}{\mathrm{~d} t^{2}}+\mu_{1} f=0
$$

if and only if there exist a nonconstant function $\phi: U^{n} \longrightarrow R$ with a negative eigenvalue $\mu_{1} \leq 0$, which is the solution of following differential equation:

$$
\Delta \phi+\mu_{1} \phi=0 .
$$

The categorization of differential equations on Riemannian manifold has become a fascinating topic of research and has been investigated by numerous researchers, for instance, [6-9].

Recently, Al-Dayel et al. [6] studied the impact of differential equation (2) on Riemannian manifold $\left(L^{n}, g\right)$ by taking the concircular vector field and proved that, under certain conditions, the Riemannian manifold $\left(L^{n}, g\right)$ is isometric to Euclidean manifold $R^{n}$. Similarly, by taking gradient conformal vector field, Chen et al. [10] identified that Riemannian manifold $\left(N^{n}, g\right)$ is isometric to the Euclidean space $R^{n}$. However, in [11], it has been proved that the complete totally real submanifold in $C P^{n}$ (complex projective space) with bounded Ricci curvature satisfying (3) is isometric to a special class of hyperbolic space. 
On the contrary, Bishop and O'Neill [12] studied the geometry of manifolds having negative curvature and confirmed that Riemannian product manifolds always have nonnegative curvature. As a result, they proposed the idea of warped product manifolds, and these manifolds are defined as follows.

Consider two Riemannian manifolds $\left(L_{1}, g_{1}\right)$ and $\left(L_{2}, g_{2}\right)$ with corresponding Riemannian metrics $g_{1}$ and $g_{2}$, and let $\psi: L_{1} \longrightarrow R$ be a positive differentiable function. If $x$ and $y$ are projection maps such that $x: L_{1} \times L_{2} \longrightarrow L_{1}$ and $y: L_{1} \times L_{2} \longrightarrow L_{2}$, which are defined as $x(m, n)=m$ and $y(m, n)=n \forall(m, n) \in L_{1} \times L_{2}$, then $L=L_{1} \times L_{2}$ is called warped product manifold if the Riemannian structure on $L$ satisfies

$$
g(\bar{E}, \bar{F})=g_{1}\left(x_{*} \bar{E}, x_{*} \bar{F}\right)+\left(\psi^{\circ} x\right)^{2} g_{2}\left(y_{*} \bar{E}, y_{*} \bar{F}\right),
$$

for all $\bar{E}, \bar{F} \in T L$. The function $\psi$ represents the warping function of $L_{1} \times L_{2}$. The Riemannian product manifold is a special case of warped product manifold in which the warping function is constant. The study of Bishop and O'Neill [12] revealed that these types of manifolds have wide range of applications in physics and theory of relativity. It is well known that the warping function is the solution of some partial differential equations, for example, Einstein field equation can be solved by the approach of the warped product [13]. The warped product is also applicable in the study of space time near to black holes [14].

Latterly, Ali et al. [7] characterized warped product submanifolds in Sasakian space form by the approach of the differential equation. The purpose of this paper is to study the impact of differential equation on skew CR-warped product submanifolds in the framework of the complex space form.

\section{Preliminaries}

Let $\bar{L}$ be an almost Hermitian manifold with an almost complex structure $J$ and a Hermitian metric $g$, i.e., $J^{2}=-I$ and $g(J X, J Y)=g(X, Y)$, for all vector fields $X, Y$ on $\bar{L}$. If the almost complex structure $J$ satisfies

$$
\left(\bar{D}_{X} J\right) Y=0 \text {, }
$$

for all $X, Y \in T \bar{L}$, where $\bar{D}$ is Levi-Civita connection on $\bar{L}$, then $(\bar{L}, J)$ is called a Kaehler manifold.

A Kaehler manifold $\bar{L}$ is called a complex space form if it has constant holomorphic sectional curvature and denoted by $\bar{L}(c)$. The curvature tensor of the complex space form $\bar{L}(c)$ is given by

$$
\begin{aligned}
\bar{R}(X, Y, Z, W)= & \frac{c}{4}[g(Y, Z) g(X, W)-g(X, Z) g(Y, W)+g(X, J Z) g(J Y, W) \\
& -g(Y, J Z) g(J X, W)+2 g(X, J Y) g(J Z, W)],
\end{aligned}
$$

for any $X, Y, Z, W \in T \bar{L}$.

Let $L$ be an $n$-dimensional Riemannian manifold isometrically immersed in a $m$-dimensional Riemannian manifold $\bar{L}$. Then, the Gauss and Weingarten formulas are $\bar{D}_{X} Y=D_{X} Y+h(X, Y)$ and $\bar{D}_{X} \xi=-A_{\xi} X+D_{X}^{\perp} \xi$, respectively, for all $X, Y \in T L$ and $\xi \in T^{\perp} L$, where $D$ is the induced Levi-Civita connection on $L, \xi$ is a vector field normal to $L, h$ is the second fundamental form of $L, D^{\perp}$ is the normal connection in the normal bundle $T^{\perp} L$, and $A_{\xi}$ is the shape operator of the second fundamental form. For any $X \in T L$ and $N \in T^{\perp} L, J X$ and $J N$ can be decomposed as follows:

$$
\begin{aligned}
J X & =P X+F X, \\
\text { and } J N & =t N+f N,
\end{aligned}
$$

where $P X$ (respectively, $t N$ ) is the tangential and $F X$ (respectively, $f N$ ) is the normal component of $J E$ (respectively, $J N)$.

It is evident that $g(\phi X, Y)=g(P X, Y)$, for any $X, Y \in T_{x} L$; this implies that $g(P X, Y)+g(X, P Y)=0$. Thus, $P^{2}$ is a symmetric operator on the tangent space $T_{x} L$, for all $x \in L$. The eigenvalues of $P^{2}$ are real and diagonalizable. Moreover, for each $x \in L$, one can observe

$$
S_{x}^{\lambda}=\operatorname{Ker}\left\{P^{2}+\mu^{2}(x) I\right\}_{x}
$$

where $I$ denotes the identity transformation on $T_{x} L$ and $\mu(x) \in[0,1]$ such that $-\mu^{2}(x)$ is an eigenvalue of $P^{2}(x)$. Furthermore, it is easy to observe that $\operatorname{Ker} F=S_{x}^{1}$ and $\operatorname{Ker} P=S_{x}^{0}$, where $S_{x}^{1}$ is the maximal holomorphic subspace of $T_{x} L$ and $S_{x}^{0}$ is the maximal totally real subspace of $T_{x} L$, and these distributions are denoted by $S^{T}$ and $S^{\perp}$, respectively. If $-\mu_{1}^{2}(x), \ldots,-\mu_{k}^{2}(x)$ are the eigenvalues of $P^{2}$ at $x$, then $T_{x} L$ can be decomposed as

$$
T_{x} L=S_{x}^{\mu_{1}} \oplus S_{x}^{\mu_{2}} \oplus \cdots \oplus S_{x}^{\mu_{k}}
$$

Every $S_{x}^{\lambda_{i}}, 1 \leq i \leq k$, is a $P$-invariant subspace of $T_{x} L$. Moreover, if $\mu_{i} \neq 0$, then $S_{x}^{\lambda_{i}}$ is even dimensional; the submanifold $L$ of a Kaehler manifold $\bar{L}$ is a generic submanifold if there exists an integer $k$ and functions $\mu_{i} 1 \leq i \leq k$ defined on $L$ with $\mu_{i} \in(0,1)$ such that

(i) Each $-\mu_{i}^{2}(x), 1 \leq i \leq k$, is a distinct eigenvalue of $P^{2}$ with

$$
T_{x} L=S_{x}^{T} \oplus S_{x}^{\perp} \oplus S_{x}^{\lambda_{1}} \oplus \cdots \oplus S_{x}^{\lambda_{k}},
$$

for any $x \in M$.

(ii) The distributions of $S_{x}^{T}, S_{x}^{\perp}$ and $S_{x}^{\mu_{i}}, 1 \leq i \leq k$, are independent of $x \in L$.

If, in addition, each $\mu_{i}$ is constant on $L$, then $L$ is called a skew CR-submanifolds [15]. It is significant to account that 
CR-submanifolds are particular class of skew CR-submanifold with $k=1, S^{T}=\{0\}, S^{\perp}=\{0\}$, and $\mu_{1}$ is constant. If $S^{\perp}=\{0\}, S_{T}^{1} \neq\{0\}$, and $k=1$, then $L$ is semislant submanifold, whereas if $S_{T}=\{0\}, S^{\perp} \neq\{0\}$, and $k=1$, then $L$ is a hemi-slant submanifold.

Definition 1. A submanifold $L$ of a Kaehler manifold $\bar{L}$ is said to be a skew CR-submanifold of order 1 if $L$ is a skew CR-submanifold with $k=1$ and $\mu_{1}$ is constant. For any orthonormal basis $\left\{e_{1}, e_{2}, \ldots, e_{n}\right\}$ of the tangent space $T_{x} L$, the mean curvature vector $\Omega(x)$ and its squared norm are defined as follows:

$$
\begin{aligned}
& \Omega(x)=\frac{1}{n} \sum_{i=1}^{n} h\left(e_{i}, e_{i}\right), \\
& \|\Omega\|^{2}=\frac{1}{n^{2}} \sum_{i, j=1}^{n} g\left(h\left(e_{i}, e_{i}\right), h\left(e_{j}, e_{j}\right)\right),
\end{aligned}
$$

where $n$ is the dimension of $L$. If $h=0$, then the submanifold is said to be totally geodesic and minimal if $\Omega=0$. If $h\left(E_{1}, E_{2}\right)=g\left(E_{1}, E_{2}\right) \Omega$, for all $E_{1}, E_{2} \in T L$, then $L$ is called totally umbilical.

The scalar curvature of $\bar{L}$ is denoted by $\bar{\tau}(L)$ and is defined as

$$
\tau(L)=\sum_{1 \leq \alpha<\beta \leq m} \kappa_{\alpha \beta},
$$

where $\kappa_{\alpha \beta}=\bar{\kappa}\left(e_{\alpha} \wedge e_{\beta}\right)$ and $m$ is the dimension of the Riemannian manifold $\vec{L}$.

Let $\left\{e_{1}, \ldots, e_{n}\right\}$ be an orthonormal basis of the tangent space $T_{x} L$, and if $e_{\gamma}$ belongs to the orthonormal basis $\left\{e_{n+1}, \ldots, e_{m}\right\}$ of the normal space $T^{\perp} L$, then we have

$$
\begin{aligned}
h_{\alpha \beta}^{\gamma} & =g\left(h\left(e_{\alpha}, e_{\beta}\right), e_{\gamma}\right), \\
\|h\|^{2} & =\sum_{\alpha, \beta=1}^{n} g h\left(e_{\alpha}, e_{\beta}\right), h\left(e_{\alpha}, e_{\beta}\right) .
\end{aligned}
$$

The global tensor field for orthonormal frame of vector field $\left\{e_{1}, \ldots, e_{n}\right\}$ on $L$ is defined as

$$
S\left(E_{1}, E_{2}\right)=\sum_{i=1}^{n}\left\{g\left(R\left(e_{i}, E_{1}\right) E_{2}, e_{i}\right)\right\},
$$

for all $E_{1}, E_{2} \in T_{x} L$, where $R$ is the Riemannian curvature tensor. The above tensor is called the Ricci tensor. If we fix a distinct vector $e_{u}$ from $\left\{e_{1}, \ldots, e_{n}\right\}$ on $L^{n}$, which is governed by $\chi$, then the Ricci curvature is defined by

$$
\operatorname{Ric}(\chi)=\sum_{\substack{\alpha=1 \\ \alpha \neq u}}^{n} \kappa\left(e_{\alpha} \wedge e_{u}\right) \text {. }
$$

A submanifold $L$ of a Kaehler manifold $\bar{L}$ is said to be skew CR-warped product submanifolds if it is warped product of the type $L=L_{1} \times_{f} L_{\perp}$, where $L_{1}$ is a semislant submanifold which was defined by N. Papaghiuc [16] and $L_{\perp}$ is a totally real submanifold. Sahin [17] proved the existence skew CR-warped product submanifolds. Recently, Ali Khan and Al-Dayel [18] studied Skew CR-warped product submanifolds of the form $L^{d}=L_{1}^{d_{1}+d_{2}} \times_{f} L_{\perp}^{d_{3}}$, where $L_{1}$ is semislant submanifold with $d_{1}$-dimensional holomorphic distribution $S$ and $d_{2}$ - dimensional slant distribution $S_{\theta}$ and $L_{\perp}^{d_{3}}$ is totally real submanifold. More precisely, they obtained Ricci curvature inequalities for these submanifolds as follows.

Theorem 1. Let $L^{d}=L_{1}^{d_{1}+d_{2}} \times{ }_{f} L_{\perp}^{d_{3}}$ be a $S$-minimal complete skew CR-warped product submanifold isometrically immersed in a Complex space form $\bar{L}^{m}(c)$. If the holomorphic and slant distributions $S$ and $S_{\theta}$ are integrable with integral submanifolds $L_{T}^{d_{1}}$ and $L_{\theta}^{d_{2}}$, respectively, then, for each orthogonal unit vector field $\chi \in T_{x} L^{d}$, either tangent to $L_{T}^{d_{1}}$ or $L_{\theta}^{d_{2}}$ or $L_{\perp}^{d_{3}}$, the Ricci curvature satisfies the following expressions.

(i) If $\chi \in T L_{T}^{d_{1}}$, then

$$
\begin{aligned}
R^{L}(\chi)+d_{3} \Delta \ln f \leq & \frac{1}{4} d^{2}\|\Omega\|^{2}+d_{3}\|\nabla \ln f\|^{2} \\
& -\frac{c}{4}\left(d-d_{1} d_{2}-d_{2} d_{3}-d_{1} d_{3}-\frac{1}{2}\right) .
\end{aligned}
$$

(ii) If $\chi \in T L_{\theta}^{d_{2}}$, then

$$
\begin{aligned}
R^{L}(\chi)+d_{3} \Delta \ln f \leq & \frac{1}{4} d^{2}\|\Omega\|^{2} \\
& +d_{3}\|\nabla \ln f\|^{2}-\frac{c}{4}\left(d-d_{1} d_{2}-d_{2} d_{3}\right. \\
& \left.-d_{1} d_{3}+1-\frac{3}{2} \cos ^{2} \theta\right) .
\end{aligned}
$$

(iii) If $\chi \in T L_{\perp}^{d_{2}}$, then

$$
\begin{aligned}
R^{L}(\chi)+d_{3} \Delta \ln f \leq & \frac{1}{4} d^{2}\|\Omega\|^{2}+d_{3}\|\nabla \ln f\|^{2} \\
& -\frac{c}{4}\left(d-d_{1} d_{2}-d_{2} d_{3}-d_{1} d_{3}+1\right),
\end{aligned}
$$

where $d_{1}, d_{2}$, and $d_{3}$ are the dimensions of $S_{T}^{d_{1}}, S_{\theta}^{d_{2}}$, and $S_{\perp}^{d_{3}}$, respectively, and $\Omega$ is the mean curvature vector.

Let $f$ be a real-valued differential function on a Riemannian manifold $L^{n}$; then, the Bochner formula [19] is stated as

$$
\frac{1}{2} \Delta|\nabla f|^{2}=R^{L}(\nabla f, \nabla f)+|H(f)|^{2}+g(\nabla \Delta f, \nabla f),
$$

where $R^{L}$ denotes Ricci tensor and $H(f)$ is the Hessian of the function $f$. 


\section{Main Results}

In this section, we obtain some characterization by the application of Bochner formula.

Theorem 2. Let $L^{d}=L_{1}^{d_{1}+d_{2}} \times{ }_{f} L_{\perp}^{d_{3}}$ be a $S$-minimalddimensional complete skew CR-warped product submanifold in a complex space form $\bar{L}^{m}(c)$, where $L_{1}$ is a semislant submanifold with $d_{1}-$ dimensional invariant distribution $S$ and $d_{2}$-dimensional slant distribution $S_{\theta}$, such that Ricci curvature $R^{L}(\chi) \geq K, K>0$. If $\chi \in S$ and satisfying the equality,

$$
\left(\lambda_{1}+d_{3}\right) K=\lambda_{1}\left[\frac{d_{3}}{d}+\frac{d^{2}}{4}\|\Omega\|^{2}-\frac{c}{4}\left(d-d_{1} d_{2}-d_{2} d_{3}-d_{1} d_{3}-\frac{1}{2}\right)\right],
$$

then the base submanifold $L_{1}^{d_{1}+d_{2}}$ is isometric to Euclidean space $R^{d_{1}+d_{2}}$, where $d_{3}$ is the dimension of the anti-invariant submanifold $L_{\perp}^{d_{3}}$ and $\lambda_{1}$ is the eigenvalue corresponding to the eigenfunction $\ln f$.

Proof. Since $\chi \in S$, by equation (16),

$$
\begin{aligned}
R^{L}(\chi)+d_{3} \Delta \ln f \leq & \frac{1}{4} d^{2}\|\Omega\|^{2}+d_{3}\|\nabla \ln f\|^{2} \\
& -\frac{c}{4}\left(d-d_{1} d_{2}-d_{2} d_{3}-d_{1} d_{3}-\frac{1}{2}\right) .
\end{aligned}
$$

By the assumption that $R_{L}(\chi) \geq K$, we have

$$
\begin{aligned}
K+d_{3} \Delta \ln f \leq & \frac{1}{4} d^{2}\|\Omega\|^{2}+d_{3}\|\nabla \ln f\|^{2} \\
& -\frac{c}{4}\left(d-d_{1} d_{2}-d_{2} d_{3}-d_{1} d_{3}-\frac{1}{2}\right) .
\end{aligned}
$$

Since $R^{L}(\chi) \geq K$, on applying the theorem of Myers [1], according to this, if Ricci curvature is greater than by a positive constant, then base manifold $L_{1}^{d_{3}}$ is compact. On integrating (22) and using Green's theorem, we obtain

$$
\begin{aligned}
\operatorname{Vol}\left(L_{1}\right) K \leq & \frac{d^{2}}{4} \int_{L_{t}}\|\Omega\|^{2} \mathrm{~d} V+d_{3} \int_{L^{d}}\|\nabla \ln f\|^{2} \mathrm{~d} V \\
& -\int_{L^{d}} \frac{c}{4}\left(d-d_{1} d_{2}-d_{2} d_{3}-d_{1} d_{3}-\frac{1}{2}\right) \mathrm{d} V,
\end{aligned}
$$

or

$$
\begin{aligned}
\int_{L^{d}}\|\nabla \ln f\|^{2} \mathrm{~d} V \geq & \frac{K}{d_{3}} \operatorname{Vol}\left(L_{1}\right)-\frac{d^{2}}{4 d_{3}} \int_{L^{d}}\|\Omega\|^{2} \mathrm{~d} V \\
& +\frac{1}{d_{3}} \int_{L^{d}} \frac{c}{4}\left(d-d_{1} d_{2}-d_{2} d_{3}-d_{1} d_{3}-\frac{1}{2}\right) \mathrm{d} V .
\end{aligned}
$$

Let $H(\ln f)$ be the Hessian of the warping function $\ln f$; then, we have

$$
|H(\ln f)-n I|^{2}=|H(\ln f)|^{2}+n^{2}|I|^{2}-2 n g(I, H(\ln f)),
$$

where $n$ is real number. The above formula provides

$$
|H(\ln f)-n I|^{2}=2 n \Delta(\ln f)+n^{2}\left(d_{1}+d_{2}\right)+|H(\ln f)|^{2} .
$$

Putting $n=\left(\lambda_{1} /\left(d_{1}+d_{2}\right)\right)$ and integrating the last equation with respect to $\mathrm{d} V$ (volume element), we obtain

$\int_{L^{d}}\left|H(\ln f)-\frac{\lambda_{1}}{d_{1}+d_{2}} I\right|^{2} \mathrm{~d} V=\int_{L^{d}}|H \ln f|^{2} \mathrm{~d} V+\int_{L^{d}} \frac{\lambda_{1}^{2}}{d_{1}+d_{2}} \mathrm{~d} V$.

Using (19), with the fact $\Delta \ln f=\lambda_{1} \ln f$, we have

$\int_{L^{d}}|H(\ln f)|^{2} \mathrm{~d} V=-\lambda_{1} \int_{L^{d}}|\nabla \ln f|^{2} \mathrm{~d} V-\int_{L^{d}} R^{L}(\nabla \ln f, \nabla \ln f)$.

Combining (27) and (28), we derive

$$
\begin{aligned}
& \int_{L^{d}}\left|H(\ln f)-\frac{\lambda_{1}}{d_{1}+d_{2}} I\right|^{2} \mathrm{~d} V \\
& =\int_{L^{d}} \frac{\lambda_{1}^{2}}{d_{1}+d_{2}} \mathrm{~d} V-\lambda_{1} \int_{L^{d}}|\nabla \ln f|^{2} \mathrm{~d} V-\int_{L^{d}} R^{L}(\nabla f, \nabla f) \mathrm{d} V .
\end{aligned}
$$

By the assumption $R^{L}(\nabla f, \nabla f) \geq K$, the above equation changes to

$$
\begin{aligned}
& \int_{L^{d}}\left|H(\ln f)-\frac{\lambda_{1}}{d_{1}+d_{2}} I\right|^{2} \mathrm{~d} V \\
& \leq \int_{L^{d}} \frac{\lambda_{1}^{2}}{d_{1}+d_{2}} \mathrm{~d} V-\lambda_{1} \int_{L^{d}}|\nabla \ln f|^{2} \mathrm{~d} V-\operatorname{KVol}\left(L_{1}\right) .
\end{aligned}
$$

Using (24), the last inequality leads to

$$
\begin{aligned}
& \int_{L^{d}}\left|H(\ln f)-\frac{\lambda_{1}}{d_{1}+d_{2}} I\right|^{2} \mathrm{~d} V \\
& \leq \int_{L^{d}} \frac{\lambda_{1}^{2}}{d_{1}+d_{2}} \mathrm{~d} V-\int_{L^{d}}\left(\frac{\lambda_{1} K}{d_{3}}+K\right) \mathrm{d} V \\
& \quad+\frac{\lambda_{1}}{d_{3}} \int_{L^{d}} \frac{c}{4}\left(d-d_{1} d_{2}-d_{2} d_{3}-d_{1} d_{3}-\frac{1}{2}\right) \mathrm{d} V \\
& \quad-\frac{\lambda_{1} d^{2}}{4 d_{3}} \int_{L^{d}}\|\Omega\|^{2} \mathrm{~d} V .
\end{aligned}
$$

If (20) holds, then the above inequality produces

$$
\left|H(\ln f)-\frac{\lambda_{1}}{d_{1}+d_{2}} I\right|^{2}=0 .
$$

Therefore, we have $H(\ln f)(X, X)=\left(\lambda_{1} /\left(d_{1}+d_{2}\right)\right)$. Hence, by the application of Tashiro's result [5], the fibre $L_{1}$ is isometric to Euclidean space $R^{d_{1}+d_{2}}$. 
If we consider the unit vector field $\chi \in T L_{\theta}^{d_{2}}$ or $\chi \in T L_{\perp}^{d_{3}}$, then we have the following results which can be proved by adopting the similar steps in Theorem 2.

Theorem 3. Let $L^{d}=L_{1}^{d_{1}+d_{2}} \times{ }_{f} L_{\perp}^{d_{3}}$ be a $S$-minimal $d$-dimensional complete skew CR-warped product submanifold in a complex space form $\bar{L}^{m}(c)$, where $L_{1}$ is a semislant submanifold with $d_{1}$-dimensional invariant distribution $S$ and $d_{2}$-dimensional slant distribution $S_{\theta}$, such that Ricci curvature $R^{L}(\chi) \geq K, K>0$. If $\chi \in S_{\theta}$ and satisfying the following equality,

$$
\left(\lambda_{1}+d_{3}\right) K=\lambda_{1}\left[\frac{d_{3}}{d}+\frac{d^{2}}{4}\|\Omega\|^{2}-\frac{c}{4}\left(d-d_{1} d_{2}-d_{2} d_{3}-d_{1} d_{3}+1-\frac{3}{2} \cos ^{2} \theta\right)\right]
$$

Then, the base submanifold $L_{1}^{d_{1}+d_{2}}$ is isometric to Euclidean space $R^{d_{1}+d_{2}}$, where $d_{3}$ is the dimension of the antiinvariant submanifold $L_{\perp}^{d_{3}}$ and $\lambda_{1}$ is the eigenvalue corresponding to the eigenfunction $\ln f$.

Theorem 4. Let $L^{d}=L_{1}^{d_{1}+d_{2}} \times{ }_{f} L_{\perp}^{d_{3}}$ be a $S$-minimalddimensional compete skew CR-warped product submanifold in a complex space form $\bar{L}^{m}(c)$, where $L_{1}$ is a semislant submanifold with $d_{1}$-dimensional invariant distribution $S$ and $d_{2}$-dimensional slant distribution $S_{\theta}$, such that Ricci curvature $R^{L}(\chi) \geq K, K>0$. If $\chi \in S_{\perp}$ and satisfying the following equality,

$$
\left(\lambda_{1}+d_{3}\right) K=\lambda_{1}\left[\frac{d_{3}}{d}+\frac{d^{2}}{4}\|\Omega\|^{2}-\frac{c}{4}\left(d-d_{1} d_{2}-d_{2} d_{3}-d_{1} d_{3}+1\right)\right]
$$

Then, the base submanifold $L_{1}^{d_{1}+d_{2}}$ is isometric to Euclidean space $R^{d_{1}+d_{2}}$, where $d_{3}$ is the dimension of the antiinvariant submanifold $L_{\perp}^{d_{3}}$ and $\lambda_{1}$ is the eigenvalue corresponding to the eigenfunction $\ln f$.

Now, we have next result which is based on the study of Garcia-Rio et al. [4].
Theorem 5. Let $L^{d}=L_{1}^{d_{1}+d_{2}} \times{ }_{f} L_{\perp}^{d_{3}}$ be a $S$-minimal complete skew CR-warped product submanifold in a complex space form $\bar{L}^{m}(c)$, where $L_{1}$ is a semislant submanifold with $d_{1}$-dimensional invariant distribution $S$ and $d_{2}$-dimensional slant distribution $S_{\theta}$, such that Ricci curvature $R^{L}(\chi)>K, K>0$. If $\chi \in S$ and satisfying the following relation,

$$
d^{2}\|\Omega\|^{2}+\frac{4\left(d_{1}+d_{2}\right) d_{3}}{\lambda_{1}}|H(\ln f)|^{2}=\frac{4\left(d_{1}+d_{2}\right) d_{3}}{\lambda_{1}}\left(\frac{c}{4}\left(d-d_{1} d_{2}-d_{2} d_{3}-d_{1} d_{3}-\frac{1}{2}\right)+K\right)
$$

for $\lambda_{1}<0$. Then, $L_{1}$ is isometric to warped product of the type $R \times{ }_{\theta} U$, where $R$ is the Euclidean line and $U$ is a complete Riemannian manifold with the warping function $\theta$, which satisfies the differential equation $\left(d \theta^{2} / d t^{2}\right)+\lambda_{1} \theta=0$.
Proof. For the warping function $\ln f$, defining the following equation on $L_{1}^{d_{1}+d_{2}}$,

$$
|K \ln f I+H(\ln f)|^{2}=K^{2}(\ln f)^{2}|I|^{2}+|H(\ln f)|^{2}+2 K(\ln f) g(I, H(\ln f)) .
$$

However, we know that $|I|^{2}=\operatorname{tr}\left(I I^{*}\right)=d_{1}+d_{2}$ and $g\left(H(\ln f), I^{*}\right)=\operatorname{tr}\left(I^{*} H(\ln f)\right)=\operatorname{tr}(H(\ln f))$; using these facts, the above equation leads to

$$
|K \ln f I+H(\ln f)|^{2}=|H(\ln f)|^{2}+\left(d_{1}+d_{2}\right) K^{2}(\ln f)^{2}-2 K \ln f \Delta \ln f .
$$


Let $\ln f$ be an eigenfunction corresponding to the eigenvalue $\lambda_{1}$ satisfying $\Delta \ln f=\lambda_{1} \ln f$, and we have $\backslash$ scale $90 \%$

$|K \ln f I+H(\ln f)|^{2}=|H(\ln f)|^{2}+\left(\left(d_{1}+d_{2}\right) K^{2}-2 K \lambda_{1}\right)(\ln f)^{2}$.
Again, using $\Delta \ln f=\lambda_{1} \ln f$, it is easy to see that

$$
\nabla \frac{(\ln f)^{2}}{2}=\ln f \lambda_{1} \ln f-|\nabla \ln f|^{2}
$$

which on integrating provides

$$
\begin{aligned}
\int_{L^{d}}(\ln f)^{2} d V= & \frac{1}{\lambda_{1}} \int_{L^{d}}|\nabla \ln f|^{2}, \\
\int_{L^{d}}|H(\ln f)+K \ln f I|^{2} \mathrm{~d} V= & \int_{L^{d}}|H(\ln f)|^{2} \mathrm{~d} V \\
& +\left(\frac{\left(d_{1}+d_{2}\right) K^{2}}{\lambda_{1}}-2 K\right) \int_{L^{d}}|\nabla \ln f|^{2} \mathrm{~d} V .
\end{aligned}
$$

Putting $K=\left(\lambda_{1} /\left(d_{1}+d_{2}\right)\right)$ in (40), we have

$$
\int_{L^{d}}\left|H(\ln f)+\frac{\lambda_{1}}{d_{1}+d_{2}} \ln f I\right|^{2} \mathrm{~d} V=\int_{L^{d}}|H(\ln f)|^{2} \mathrm{~d} V-\frac{\lambda_{1}}{d_{1}+d_{2}} \int_{L^{d}}|\nabla \ln f|^{2} \mathrm{~d} V .
$$

Furthermore, integrating (16) and applying Green's Lemma, we find

$$
\int_{L^{d}} R^{L}(\chi) \mathrm{d} V \leq \frac{d^{2}}{4} \int_{L^{d}}\|\Omega\|^{2} \mathrm{~d} V+d_{3} \int_{L^{d}}\|\nabla \ln f\|^{2} \mathrm{~d} V-\int_{L^{d}} \frac{c}{4}\left(d-d_{1} d_{2}-d_{2} d_{3}-d_{1} d_{3}-\frac{1}{2}\right) \mathrm{d} V .
$$

From the above two expressions, we have

$$
\begin{aligned}
\frac{1}{d_{3}} \int_{L^{d}} R^{L}(\chi) \mathrm{d} V \leq & \frac{d^{2}}{4 d_{3}} \int_{L^{d}}\|\Omega\|^{2} \mathrm{~d} V+\frac{d_{1}+d_{2}}{\lambda_{1}} \int_{L^{d}}|H(\ln f)|^{2} \mathrm{~d} V-\frac{d_{1}+d_{2}}{\lambda_{1}} \int_{L^{d}}\left|H(\ln f)+\frac{\lambda_{1}}{d_{1}+d_{2}} \ln f I\right|^{2} \mathrm{~d} V \\
& -\int_{L^{d}} \frac{c}{4}\left(d-d_{1} d_{2}-d_{2} d_{3}-d_{1} d_{3}-\frac{1}{2}\right) \mathrm{d} V .
\end{aligned}
$$

On using the assumption that $R^{L}(\chi) \geq K$, for $K>0$,

$$
\begin{aligned}
\int_{L^{d}}\left|H(\ln f)+\frac{\lambda_{1}}{d_{1}+d_{2}} \ln f I\right|^{2} \mathrm{~d} V \leq & \frac{d^{2} \lambda_{1}}{4\left(d_{1}+d_{2}\right) d_{3}} \int_{L^{d}}\|\Omega\|^{2} \mathrm{~d} V+\int_{L^{d}}|H(\ln f)|^{2} \mathrm{~d} V-\frac{\lambda_{1}}{\left(d_{1}+d_{2}\right) d_{3}} \int_{L^{d}} K \mathrm{~d} V \\
& -\frac{\lambda_{1}}{d_{1}+d_{2}} \int_{L^{d}} \frac{c}{4}\left(d-d_{1} d_{2}-d_{2} d_{3}-d_{1} d_{3}-\frac{1}{2}\right) \mathrm{d} V .
\end{aligned}
$$

Equivalently, 


$$
\int_{L^{d}}\left|H(\ln f)+\frac{\lambda_{1}}{d_{1}+d_{2}} \ln f I\right|^{2} \mathrm{~d} V \leq \int_{L^{d}}\left\{\frac{\lambda_{1}}{d_{1}+d_{2}}\left(\frac{d^{2}}{4 d_{3}}\|\Omega\|^{2}-\frac{c}{4}\left(d-d_{1} d_{2}-d_{2} d_{3}-d_{1} d_{3}-\frac{1}{2}\right)\right)+\frac{K}{d_{3}}+|H(\ln f)|^{2}\right\} \mathrm{d} V .
$$

By assumption (35), we obtain

$$
d^{2}\|\Omega\|^{2}+\frac{4\left(d_{1}+d_{2}\right) d_{3}}{\lambda_{1}}|H(\ln f)|^{2}=\frac{4\left(d_{1}+d_{2}\right) d_{3}}{\lambda_{1}}\left(\frac{c}{4}\left(d-d_{1} d_{2}-d_{2} d_{3}-d_{1} d_{3}-\frac{1}{2}\right)+\frac{K}{d_{3}}\right) .
$$

In view of (45) and (46), we find

$$
\begin{aligned}
& \left|H(\ln f)+\frac{\lambda_{1}}{d_{1}+d_{2}} \ln f I\right|^{2} \leq 0, \\
& \text { or } H(\ln f)+\frac{\lambda_{1}}{d_{1}+d_{2}} \ln f I=0 .
\end{aligned}
$$

By taking trace of the above equation, we obtain

$$
\Delta \ln f+\lambda_{1} \ln f=0 .
$$

Now, by the application of the result obtained in [4], together with the fact that $L^{d}=L_{1}^{d_{1}+d_{2}} \times{ }_{f} L_{\perp}^{d_{3}}$ is nontrivial, we deduced that $L_{1}$ is isometric to a warped product of the form $R \times{ }_{\theta} U$, where $U$ is complete Riemannian manifold. In addition, the warping function $\theta$ is the solution of the differential equation $\left(\mathrm{d} \theta^{2} / \mathrm{d} t^{2}\right)+\lambda_{1} \theta=0$. Hence, the proof is completed.

Similarly, we can prove the following theorems by taking the unit vector field $\chi$ tangent to $L_{\theta}^{d_{2}}$ and $L_{\perp}^{d_{3}}$, respectively.

Theorem 6. Let $L^{d}=L_{1}^{d_{1}+d_{2}} \times{ }_{f} L_{\perp}^{d_{3}}$ be a $S$-minimal complete skew CR-warped product submanifold in a complex space form $\bar{L}^{m}(c)$, where $L_{1}$ is a semislant submanifold with $d_{1}$-dimensional invariant distribution $S$ and $d_{2}$-dimensional slant distribution $S_{\theta}$, such that Ricci curvature $R^{L}(\chi)>K, K>0$. If $\chi \in S_{\theta}$ and satisfying the following relation,

$$
d^{2}\|\Omega\|^{2}+\frac{4\left(d_{1}+d_{2}\right) d_{3}}{\lambda_{1}}|H(\ln f)|^{2}=\frac{4\left(d_{1}+d_{2}\right) d_{3}}{\lambda_{1}}\left(\frac{c}{4}\left(d-d_{1} d_{2}-d_{2} d_{3}-d_{1} d_{3}+1-\frac{3}{2} \cos ^{2} \theta\right)+K\right)
$$

for $\lambda_{1}<0$. Then, $L_{1}$ is isometric to warped product of the type $R \times{ }_{\theta} U$, where $R$ is the Euclidean line and $U$ is a complete Riemannian manifold with the warping function $\theta$, which satisfies the differential equation $\left(d \theta^{2} / d t^{2}\right)+\lambda_{1} \theta=0$.

Theorem 7. Let $L^{d}=L_{1}^{d_{1}+d_{2}} \times_{f} L_{\perp}^{d_{3}}$ be a $S$-minimal complete Skew CR-warped product submanifold in a complex space form $\bar{L}^{m}(c)$, where $L_{1}$ is a semislant submanifold with $d_{1}$-dimensional invariant distribution $S$ and $d_{2}$-dimensional slant distribution $S_{\theta}$, such that Ricci curvature $R^{L}(\chi)>K, K>0$. If $\chi \in S_{\perp}$ and satisfying the following relation,

$$
d^{2}\|\Omega\|^{2}+\frac{4\left(d_{1}+d_{2}\right) d_{3}}{\lambda_{1}}|H(\ln f)|^{2}=\frac{4\left(d_{1}+d_{2}\right) d_{3}}{\lambda_{1}}\left(\frac{c}{4}\left(d-d_{1} d_{2}-d_{2} d_{3}-d_{1} d_{3}+1\right)+K\right),
$$

for $\lambda_{1}<0$. Then, $L_{1}$ is isometric to warped product of the type $R \times{ }_{\theta} U$, where $R$ is the Euclidean line and $U$ is a complete Riemannian manifold with the warping function $\theta$, which satisfies the differential equation $\left(d \theta^{2} / d t^{2}\right)+\lambda_{1} \theta=0$.

\section{Conclusions}

This paper studies the geometric behavior of ordinary differential equations on the skew CR-warped product submanifolds. More precisely, we obtain characterizing theorems for skew CR-warped product submanifolds of complex space forms via differential and integral theory on
Riemannian manifolds. Therefore, the present article provides a wonderful correlation of theory of differential equations with the warped product submanifolds.

\section{Data Availability}

No data were used to support the findings of the study.

\section{Conflicts of Interest}

The authors declare that they have no conflicts of interest. 


\section{References}

[1] S. B. Myers, "Riemannian manifolds with positive mean curvature," Duke Mathematical Journal, vol. 8, no. 2, pp. 401-404, 1941.

[2] S. Tanno, "Some differential equations on Riemannian manifolds," Journal of the Mathematical Society of Japan, vol. 30, no. 3, pp. 509-531, 1978.

[3] S. Deshmukh, "Characterizing spheres and Euclidean spaces by conformal vector fields," Annali di Matematica Pura ed Applicata (1923), vol. 196, no. 6, pp. 2135-2145, 2017.

[4] E. Garcia-Rio, D. N. Kupeli, and B. Unal, "Approximation for the wave equation in a moving domain," Control of Partial Differential Equations, 1994, pp. 287-295, 2003.

[5] Y. Tashiro, "Complete Riemannian manifolds and some vector fields," Transactions of the American Mathematical Society, vol. 117, p. 251, 1965.

[6] I. Al-Dayel, S. Deshmukh, and O. Belova, "A remarkable property of concircular vector fields on a Riemannian manifold," Mathematics, vol. 8, no. 4, p. 469, 2020.

[7] A. Ali, F. Mofarreh, W. A. Mior Othman, and D. S. Patra, "Applications of differential equations to characterize the base of warped product submanifolds of cosymplectic space forms," Journal of Inequalities and Applications, vol. 2020, no. 1, p. $241,2020$.

[8] R. Ali, F. Mofarreh, N. Alluhaibi, A. Ali, and I. Ahmad, "On differential equations characterizing Legendrian submanifolds of Sasakian space forms," Mathematics, vol. 8, no. 2, p. 150, 2020.

[9] M. Jamali and M. H. Shahid, "Application of Bochner formula to generalized Sasakian space forms," Africa Mathematics, vol. 29, pp. 1135-1139, 2018.

[10] B.-Y. Chen, S. Deshmukh, and A. A. Ishan, "On Jacobi-type vector fields on Riemannian manifolds," Mathematics, vol. 7, no. 12, p. 1139, 2019.

[11] Y. Matsuyama, "Complete totally real submanifolds of a complex projective space," Differential Geometry-Dynamical Systems, vol. 20, pp. 119-125, 2018.

[12] R. L. Bishop and B. O'Neill, "Manifolds of negative curvature," Transactions of the American Mathematical Society, vol. 145, pp. 1-91, 1969.

[13] J. K. Beem, P. Ehrlich, and T. G. Powell, Warped Product Manifolds in Relativity Selected Studies, North-Holland, Amsterdam, NY, USA, 1982.

[14] S. W. Hawkings and G. F. R. Ellis, The Large Scale Structure of Space-Time, Cambridge Univercity Press, Cambridge, UK, 1973.

[15] G. S. Ronsse, "Generic and skew CR-submanifolds of a Kaehler manifold," Bulletin of the Institute of Mathematics Academia Sinica, vol. 18, pp. 127-141, 1990.

[16] N. Papaghiuc, "Semi-slant submanifolds of Kaehler manifolds, Analele. stiintifice ale Universitatii Al. I," Cuza Din Iasi, vol. 40, pp. 55-61, 1994.

[17] B. Sahin, "Skew CR-warped products of Kaehler manifolds," Mathematical Communications, vol. 15, pp. 189-204, 2010.

[18] M. Ali Khan and I. Aldayel, "Ricci curvature inequalities for skew CR-warped product submanifolds in complex space forms," Mathematics, vol. 8, no. 8, p. 1317, 2020.

[19] M. Berger, "Les varietes riemanniennes (1/4)-pinces," Annals of Science Normal Superiore the Pisa Class Science, vol. 14, no. 2, pp. 161-170, 1960. 